\title{
WEAKLY COERCIVE MAPPINGS SHARING A VALUE
}

\author{
J. M. Soriano, Sevilla
}

(Received July 7, 2009)

\begin{abstract}
Some sufficient conditions are provided that guarantee that the difference of a compact mapping and a proper mapping defined between any two Banach spaces over $\mathbb{K}$ has at least one zero. When conditions are strengthened, this difference has at most a finite number of zeros throughout the entire space. The proof of the result is constructive and is based upon a continuation method.
\end{abstract}

Keywords: zero point, continuation method, $C^{1}$-homotopy, surjerctive implicit function theorem, proper mapping, compact mapping, coercive mapping, Fredholm mapping

MSC 2010: 58C30, 65H10

\section{Preliminaires}

Let $X$ and $Y$ be two Banach spaces. If $u: D(F) \subseteq X \rightarrow Y$ is a continuous mapping, then one way of solving the equation

$$
u(x)=0
$$

is to embed (1) in a continuum of problems

$$
H(x, t)=0 \quad(0 \leqslant t \leqslant 1)
$$

which can easily be resolved when $t=0$. When $t=1$, the problem (2) becomes (1). In the case when it is possible to continue the solution for all $t$ in $[0,1]$ then (1) is solved. This method is called continuation with respect to a parameter [1]-[9].

In this paper some sufficient conditions are provided in order to guarantee that the difference of a compact and a proper weakly coercive $C^{1}$-mapping has at least

This work is partially supported by D.G.E.S. Pb 96-1338-CO 2-01 and the Junta de Andalucia. 
one zero. If these conditions are conveniently strengthened this difference has at most a finite number of zeros on $X$. Other conditions, sufficient to guarantee the existence of fixed points, have been given by the author in a finite-dimensional setting, see for example [7], and in an infinite-dimensional setting, see for example [8]. A continuation method was used in the proofs of these papers. The proofs supply the existence of implicitly defined continuous mappings whose ranges reach zero points [1]-[9]. A continuation method is also used here. The key is the use of the surjective implicit function theorem [10], and the properties of proper and Fredholm $C^{1}$-mappings (see [9]).

We briefly recall some theorems and concepts to be used.

Definitions [26], [27]. Henceforth we will assume that $X$ and $Y$ are Banach spaces over $\mathbb{K}$, where $\mathbb{K}=\mathbb{R}$ or $\mathbb{K}=\mathbb{C}$.

A mapping $F: D(F) \subseteq X \rightarrow Y$ is called weakly coercive if and only if $\|F(x)\| \rightarrow$ $\infty$ as $\|x\| \rightarrow \infty$.

A mapping $F: D(F) \subseteq X \rightarrow Y$ is said to be compact whenever it is continuous and the image $F(B)$ is relatively compact (i.e. its closure $\overline{F(B)}$ is compact in $Y$ ) for every bounded subset $B \subset D(F)$.

A mapping $F$ is said to be proper whenever the pre-image $F^{-1}(K)$ of every compact subset $K \subset Y$ is also a compact subset of $D(F)$.

The symbol dim means dimension, codim means codimension, ker means kernel, $R(L)$ stands for the range of the mapping $L$.

That $L: X \rightarrow Y$ is a linear Fredholm mapping means that $L$ is linear and continuous and both the numbers $\operatorname{dim}(\operatorname{ker}(L))$ and $\operatorname{codim}(R(L))$ are finite, and therefore $\operatorname{ker}(L)=X_{1}$ is a Banach space and has the topological complement $X_{2}$, since $\operatorname{dim}\left(X_{1}\right)$ is finite. The integer number $\operatorname{Ind}(L)=\operatorname{dim}(\operatorname{ker}(L))-\operatorname{codim}(R(L))$ is called the index of $L$.

Let $F: D(F) \subseteq X \rightarrow Y$. If $D(F)$ is open, then the mapping $F$ is said to be a Fredholm mapping if and only if $F$ is a $C^{1}$-mapping and $F^{\prime}(x): X \rightarrow Y$ is a Fredholm linear mapping for all $x \in D(F)$. If $\operatorname{Ind}\left(F^{\prime}(x)\right)$ is constant with respect to $x \in D(F)$, then we call this number the index of $F$ and write it as $\operatorname{Ind}(F)$.

$X, Y$ are called isomorphic if and only if there is a linear homeomorphism (isomorphism) $L: X \rightarrow Y$.

Let $\mathcal{F}(X, Y)$ denote the set of all linear Fredholm mappings $A: X \rightarrow Y$.

Let $\mathcal{L}(X, Y)$ denote the set of all linear continuous mappings $L: X \rightarrow Y$.

Let $\operatorname{Isom}(X, Y)$ denote the set of all isomorphisms $L: X \rightarrow Y$.

Let $F: D(F) \subseteq X \rightarrow Y$ with $D(F)$ open be a $C^{1}$-mapping. The point $u \in X$ is called a regular point of $F$ if and only if $F^{\prime}(u) \in \mathcal{L}(X, Y)$ maps onto $Y$. A point 
$v \in Y$ is called a regular value of $F$ if and only if the pre-image $F^{-1}(v)$ is empty or consists solely of regular points.

Theorem 1. The Surjective Implicit Function Theorem. [10, Section 4-13, Theorem $4-\mathrm{H}]$. Let $X, Y, Z$ be Banach spaces over $\mathbb{K}=\mathbb{R}$ or $\mathbb{K}=\mathbb{C}$, and let

$$
F: U\left(u_{0}, v_{0}\right) \subseteq X \times Y \rightarrow Z
$$

be a $C^{1}$-mapping on an open neighbourhood of the point $\left(u_{0}, v_{0}\right)$. Suppose that

(i) $F\left(u_{0}, v_{0}\right)=0$, and

(ii) $F_{v}\left(u_{0}, v_{0}\right): Y \rightarrow Z$ is surjective.

Then the following assertion is true:

Let $r>0$. There is a number $\varrho>0$ such that, for each given $u \in X$ with $\left\|u-u_{0}\right\|<\varrho$, the equation

$$
F(u, v)=0
$$

has a solution $v$ such that $\left\|v-v_{0}\right\|<r$.

Theorem 2 [9, Section 7-9, Theorem 7-33]. Let $g: D(g) \subseteq X \rightarrow Y$ be a compact mapping, where $a \in D(g)$ and $D(g)$ is open. If the derivative $g^{\prime}(a)$ exists, then $g^{\prime}(a) \in \mathcal{L}(X, Y)$ is also a compact mapping.

Theorem $3[9$, Section 8-4, Example 8-16]. Let $S \in \mathcal{F}(X, Y)$. The perturbed mapping $S+C$ verifies $S+C \in \mathcal{F}(X, Y)$ and $\operatorname{Ind}(S+C)=\operatorname{Ind}(S)$ provided $C \in$ $\mathcal{L}(X, Y)$ and $C$ is a compact mapping.

\section{WEAKLY COERCIVE MAPPINGS SHARING A VALUE}

Clearly, if we define $u:=f-g$, then $u$ has a zero if and only if $f$ and $g$ share a value, that is, there is $x \in X$ with $f(x)=g(x)$. We thereby establish our result in terms of $f, g$.

Theorem 4. Let $f, g: D \subseteq X \rightarrow Y$ be two $C^{1}$-mappings, where $X$ and $Y$ are two Banach spaces over $\mathbb{K}=\mathbb{R}$ or $\mathbb{K}=\mathbb{C}$, and $D$ is open.

We assume

(i) $f$ is a compact mapping, $g$ is a proper mapping and $t f(x)-g(x)$ is weakly coercive, jointly in both coordinates.

(ii) The mapping $g$ has a zero, $x_{0}$.

(iii) For any fixed $t$, belonging to $[0,1]$, the zero of $Y$ is a regular value of the mapping $t f(x)-g(x)$. 
Then the following assertion holds

(a) $f$ and $g$ share at least one value, i.e., there is at least one $x^{*}$ such that $f\left(x^{*}\right)=g\left(x^{*}\right)$.

If in addition the condition

(iv) $g$ is a Fredholm mapping of index zero is satisfied, then in addition we have

(b) $f$ and $g$ share at most a finite number of values on $D$, and at least one value.

Proof.

(a) Conclusion (a) will be proved in this section.

(a1) Let us define a mapping

$$
H: D \times[0,1] \subseteq X \times[0,1] \rightarrow Y, \text { where } H(x, t):=t f(x)-g(x)
$$

We will prove here that $H$ is a proper mapping, which will imply that $H^{-1}(0)$ is a compact set, since $\{0\} \subset Y$ is a compact set and $H$ is proper.

Let $\mathcal{C}$ be any fixed compact subset of $Y$, and let a sequence be fixed such that $\left(H\left(x_{n}, t_{n}\right)\right)_{n \geqslant 1}$ belongs to $\mathcal{C}$. It suffices to show that the sequence $\left(\left(x_{n}, t_{n}\right)\right)_{n \geqslant 1}$ contains a convergent subsequence $\left(\left(x_{n^{\prime \prime \prime}}, t_{n^{\prime \prime \prime}}\right)\right)_{n^{\prime \prime \prime} \geqslant 1}$, which will imply that $H^{-1}(\mathcal{C})$ is relatively compact, and since

$$
\left(x_{n^{\prime \prime \prime}}, t_{n^{\prime \prime \prime}}\right) \rightarrow(u, t) \quad \text { as } \quad n^{\prime \prime \prime} \rightarrow \infty
$$

$H$ is continuous and $\mathcal{C}$ compact, therefore $H(u, t) \in \mathcal{C}$, that is, $(u, t) \in H^{-1}(\mathcal{C})$, and hence $H^{-1}(\mathcal{C})$ is compact.

Since the set $\mathcal{C}$ is bounded and the mapping $H$ is weakly coercive, $\left(\left(x_{n}, t_{n}\right)\right)_{n \geqslant 1}$ is a bounded sequence. Consequently $\left(x_{n}\right)_{n \geqslant 1}$ and $\left(t_{n}\right)_{n \geqslant 1}$ are bounded sequences. Since $f$ is a compact mapping and $\left(x_{n}\right)_{n \geqslant 1}$ a bounded sequence, there exists a subsequence $\left(x_{n^{\prime}}\right)_{n^{\prime} \geqslant 1}$ such that

$$
f\left(x_{n^{\prime}}\right) \rightarrow w^{\prime} \quad \text { as } \quad n^{\prime} \rightarrow \infty
$$

for some $w^{\prime} \in Y$, and furthermore, since $\left(t_{n}\right)_{n \geqslant 1}$ is a bounded sequence of real numbers, there is $t \in \mathbb{R}$ with

$$
t_{n^{\prime}} \rightarrow t \quad \text { as } \quad n^{\prime} \rightarrow \infty
$$

Therefore

$$
t_{n^{\prime}} f\left(x_{n^{\prime}}\right) \rightarrow t w^{\prime}:=w \quad \text { as } \quad n^{\prime} \rightarrow \infty
$$

for some $w \in Y$. 
All $H\left(x_{n^{\prime}}, t_{n^{\prime}}\right), n^{\prime} \geqslant 1$ lie in the compact set $\mathcal{C}$ and therefore, there is a subsequence $\left(H\left(x_{n^{\prime \prime}}, t_{n^{\prime \prime}}\right)\right)_{n^{\prime \prime} \geqslant 1}$ such that

$$
H\left(x_{n^{\prime \prime}}, t_{n^{\prime \prime}}\right) \rightarrow v \quad \text { as } \quad n^{\prime \prime} \rightarrow \infty
$$

for some $v \in \mathcal{C}$. Therefore

$$
g\left(x_{n^{\prime \prime}}\right) \rightarrow w-v \quad \text { as } \quad n^{\prime \prime} \rightarrow \infty
$$

Since $g$ is proper, $\left(x_{n^{\prime \prime}}\right)$ has a convergent subsequence

$$
x_{n^{\prime \prime \prime}} \rightarrow u \quad \text { as } \quad n^{\prime \prime} \rightarrow \infty .
$$

On the other hand,

$$
t_{n^{\prime \prime \prime}} \rightarrow t \quad \text { as } \quad n^{\prime \prime \prime} \rightarrow \infty
$$

hence

$$
\left(x_{n^{\prime \prime \prime}}, t_{n^{\prime \prime \prime}}\right) \rightarrow(u, t) \quad \text { as } \quad n^{\prime \prime \prime} \rightarrow \infty
$$

as required.

(a2) Let us suppose that

$$
H\left(x_{a}, t_{a}\right)=0
$$

for a fixed $\left(x_{a}, t_{a}\right)$. We will prove that there is $\varrho>0$ such that for any $t \in\left(t_{a}-\varrho\right.$, $\left.t_{a}+\varrho\right)$, there exists $x=x(t)$ such that $H(x(t), t)=0$.

Since zero is a regular value for the mappings $\{t f(x)-g(x)\}$ by hypothesis (iii),

$$
H_{x}(x, t)=t f^{\prime}(x)-g^{\prime}(x) \in \mathcal{L}(X, Y)
$$

is surjective for every pair $(x, t)$ such that $H(x, t)=0$. In particular, the mapping

$$
H_{x}\left(x_{a}, t_{a}\right)=t_{a} f^{\prime}\left(x_{a}\right)-g^{\prime}\left(x_{a}\right) \in \mathcal{L}(X, Y)
$$

is surjective, which together with identity (3) and Theorem 1 implies the existence of $\varrho>0, r>0$ such that for any $t \in\left(t_{a}-\varrho, t_{a}+\varrho\right)$ there is $x(t)$ with $H(x(t), t)=0$ and $\left\|x(t)-x_{a}\right\|<r$.

(a3) We will prove that for every $t$ in $[0,1]$ there exists $x(t)$ such that $H(x(t), t)=0$.

Let $M$ denote the set of all $t$ such that there is a solution $x(t)$. By assumption (ii) this set is not empty. By (a2), the set is relatively open. Finally, since the set is the projection of $H^{-1}(0)$ into the second component (i.e. the $t$ component), and since the set is the image of a compact set by the continuous function projection, 
it is therefore compact, and hence also closed. However, a relatively open, closed, non-empty subset of $[0,1]$ is the whole interval, since $[0,1]$ is connected.

Thus

$$
H(x(1), 1)=f(x(1))-g(x(1))=0 \Rightarrow f(x(1))=g(x(1)),
$$

which is conclusion (a) of the theorem, where $x^{*}:=x(1)$.

(b) We include the hypothesis (iv) in Section (b).

(b1) We will see that the $C^{1}$-mapping $u: D \subseteq X \rightarrow Y, u:=f-g$ is a proper Fredholm mapping of index zero.

Since $f$ is a compact mapping, Theorem 2 implies that for each $x \in X$, the mapping $f^{\prime}(x) \in \mathcal{L}(X, Y)$ is also a compact mapping.

Since $f^{\prime}(x)$ is a linear compact mapping and given that $g^{\prime}(x)$ is a linear Fredholm mapping of index zero, Theorem 3 implies that

$$
u^{\prime}(x)=f^{\prime}(x)-g^{\prime}(x) \in \mathcal{L}(X, Y), \quad \forall x \in D
$$

is a Fredholm linear mapping and $\operatorname{Ind}\left(u^{\prime}(x)\right)=\operatorname{Ind}\left(g^{\prime}(x)\right)=0, \forall x \in D$. Therefore the non-linear $C^{1}$-mapping $u$ is a Fredholm mapping of index zero.

Furthermore, Section (a1) implies that $u$ is a proper mapping. In fact, this is the particular case in which $t=1$, i.e. $u(x)=H(x, 1)$.

(b2) We will see that, if any $x \in D$ exists which verifies $u(x)=0$, then $u$ is a local $C^{1}$-diffeomorphism at $x$.

Let $x \in D$ exist such that $u(x)=0$. Since zero is a regular value of $u$, the linear Fredhom mapping $u^{\prime}(x)$ maps onto $Y$, and since $\operatorname{Ind}\left(u^{\prime}(x)\right)=0$, therefore $\operatorname{dim}\left(\operatorname{ker}\left(u^{\prime}(x)\right)\right)=0$. Thus $u^{\prime}(x) \in \operatorname{Isom}(X, Y)$. By the Local Inverse Theorem [10], $u$ is a local diffeomorphism at $x$.

(b3) We will prove here that $f$ and $g$ share at most a finite number of values on $D$.

Since $u$ is proper, $u^{-1}(0)$ is a compact set. If there were an infinite sequence

$$
\left(x_{n}\right)_{n \geqslant 1} \subset D \quad \text { with } x_{n} \neq x_{m} \text { when } n \neq m
$$

verifying $u\left(x_{n}\right)=0, \forall n \in \mathbb{N}$, there would be a subsequence $\left(x_{n^{\prime}}\right)_{n^{\prime} \geqslant 1} \subset u^{-1}(0)$, which would converge at a point $x \in u^{-1}(0)$, and $x$ would be an non-isolated zero of $u$. However, since $u$ is a local diffeomorphism at $x$, given in Section (b2), $x$ is an isolated zero of $u$. This is a contradiction. Hence there is not an infinite number of zeros of $u$ on $D$. Thus $f$ and $g$ share at most a finite number of values on $D$.

Example. Let us consider an integral mapping

$$
(A u)(x)=\int_{a}^{b} F(x, y, u(y)) \mathrm{d} y, \quad \forall x \in[a, b],
$$


where $-\infty<a<b<+\infty$. Define

$$
Q:=\left\{(x, y, u) \in \mathbb{R}^{3}: x, y \in[a, b] \text { and }|u|<r \text { for fixed } r>0\right\}
$$

Suppose that the function

$$
F:\left\{(x, y, u) \in \mathbb{R}^{3}: x, y \in[a, b] \text { and }|u| \leqslant r\right\} \rightarrow \mathbb{R}
$$

is twice continuously differentiable. Define $X:=C[a, b]$ and $M:=\{u \in X$ : $\|u\|<r\}$, where $\|u\|=\max _{a \leqslant y \leqslant b}|u(y)|$. It can be easily proved that the mapping $A: M \rightarrow X$ is compact, and it is twice cotinuously differentiable.

Let $B$ be the mapping $B: M \times[0,1] \rightarrow X, B(u, t)=u$ which is $C^{\infty}(M \times[0,1], X)$, is proper, has a zero, and since it is defined only on a bounded set, it is trivially weakly coercive.

The mapping $H(u, t)=t(A u)-(B u): M \times[0,1] \rightarrow X$ is weakly coercive, since it is defined only on a bounded set. Theorem 4 implies that if zero is a regular value, then there is $u \in M$ such that

$$
(A u)(x)=(B u)(x), \forall x \in[a, b]
$$

If we do not know that zero is a regular value, it is possible to prove the existence of $u \in M$ with $(A u)(x)$ as near to $u(x), \forall x \in[a, b]$ as wanted in the following way:

Since $B^{\prime}(u, t) \in \mathcal{L}(X \times \mathbb{R}, X)$ is surjective and $\operatorname{dim}\left(\operatorname{ker}\left(B^{\prime}(u, t)\right)\right)=1$ for $\forall(u, t) \in$ $M \times[0,1], B$ is a Fredholm mapping of index one.

Define a mapping $A^{*}: M \times[0,1] \rightarrow X, A^{*}(u, t):=t(A u)$ that is differentiable and compact. Theorem 2 implies that $\left(A^{*}\right)^{\prime}(u, t)$ is compact. Theorem 3 implies that $H^{\prime}(u, t)$ is a linear Fredholm mapping of index one for $\forall(u, t) \in M \times[0,1]$, and hence $H(u, t)$ is a Fredholm mapping of index one. Thus we obtain from the Sard-Smale theorem [9, Theorem $4 . \mathrm{K}]$ that the set of regular values of the proper mapping $H$ is open and dense in $X$.

Acknowledgment. I am deeply indebted to the referee for helpful suggestions. 


\section{References}

[1] E. L. Allgower: A survey of homotopy methods for smooth mappings. Allgower, Glashoff, and Peitgen (eds.) Springer-Verlag, Berlin, 1981, pp. 2-29.

[2] E. L. Allgower, K. Glashoff and H. Peitgen (eds.): Proceedings of the Conference on Numerical Solution of Nonlinear Equations. Bremen, July 1980, Lecture Notes in Math. 878. Springer-Verlag, Berlin, 1981.

[3] E. L. Allgower and K. Georg: Numerical Continuation Methods. Springer Series in Computational Mathematics 13, Springer-Verlag, New York, 1990.

[4] J. C. Alexander and J. A. York: Homotopy Continuation Method: numerically implementable topological procedures. Trans. Amer. Math. Soc. 242 (1978), 271-284.

[5] C. B. Garcia and T. I. Li: On the number of solutions to polynomial systems of non-linear equations. SIAM J. Numer. Anal. 17 (1980), 540-546.

[6] C.B. Garcia and W.I. Zangwill: Determining all solutions to certain systems of nonlinear equations. Math. Operations Research 4 (1979), 1-14.

[7] J. M. Soriano: Global minimum point of a convex function. Appl. Math. Comput. 55 (1993), 213-218.

[8] J. M. Soriano: Continuous embeddings and continuation methods. Nonlinear Anal. Theory Methods Appl. 70 (2009), 4118-4121.

[9] E. Zeidler: Nonlinear Functional Analysis and its applications I. Springer-Verlag, New York, 1992.

[10] E. Zeidler: Applied Functional Analysis. Springer-Verlag, Applied Mathematical Sciences 109, New York, 1995.

Author's address: J. M. Soriano, Departamento de Análisis Matemático, Facultad de Matemáticas, Universidad de Sevilla, Aptdo. 1160, Sevilla 41080, Spain, e-mail: soriano@us.es. 